\title{
Emerging Opportunities in STEM to Characterize Soft-Hard Interfaces
}

Stephanie Ribet ${ }^{1}$, Akshay Murthy $^{2}$, Eric Roth ${ }^{3}$, Xiaobing $\mathrm{Hu}^{4}$, Roberto dos Reis ${ }^{4}$ and Vinayak Dravid ${ }^{4}$

${ }^{1}$ Evanston, Illinois, United States, ${ }^{2}$ Department of Materials Science, Northwestern University, United States, ${ }^{3}$ NUANCE Center, Northwestern University, United States, ${ }^{4}$ Department of Materials Science and Engineering, Northwestern University, United States

Inspired by the architectures composed of hard and soft materials in natural systems, synthetic hybrid structures have evoked significant interest in scientific and engineering communities. The dichotomy presented by the disparate physical properties of the hard and soft components offers considerable opportunities for novel architectures in a wide range of applications.[1,2] As the nature of the interface bridging the hard and soft materials often dictates the resultant properties and unique phenomena in these structures, the study of these junctions is important for both natural and engineered hybrid systems.[3]

Scanning transmission electron microscopy (STEM) is a natural choice for characterizing the interfaces present in these hybrid materials. As a multimodal technique, STEM allows for concurrent probing of structures and properties at the molecular and nano length scales relevant for understanding the role interfaces play in these systems.

Nonetheless, it is well known that applying STEM techniques is not straightforward for these hybrid structures for a variety of reasons. Careful sample preparation is necessary to preserve the interfacial regions in these materials due to the disparate mechanical properties present in adjacent components. Moreover, due to large discrepancies in the scattering cross-section of heavy and light elements, it can be intrinsically difficult to obtain appreciable signal from both hard and soft components using traditional methods. Finally, the most beam sensitive material puts an upper limit on the allowable dose, limiting the obtainable signal to noise from all components.

Emerging advances across the entire microscopy workflow, including sample preparation and data transfer have made it more feasible to interrogate critical interfacial regions using STEM.[4] Here we demonstrate two examples of how specialized data collection can be applied to understand hybrid interfaces.

It has been demonstrated that introducing a phase [5] or amplitude [6] plate into the probe forming aperture of a STEM leads to improved transfer of information at low spatial frequencies, especially when paired with intelligent virtual detector design. Using the archetypical Fresnel zone plate, we employ this strategy to a simulated molybdenum ditelluride-graphene layered heterostructure. Figure 1 shows the (a) control and (b) modified probe, where opposing rings are shifted by 0 and $\pi / 2$. We calculate the (c) contrast transfer function of the modified probe, which shows high information transfer at low spatial frequencies. Comparing the (d) projected potential, (e) conventional ADF, and (f) phase plate/virtual detector image, it is clear that the signal from all elements is significantly enhanced with a phase plate configuration.

An ADF image of a hybrid structure composed of iron oxide (hard), gold (hard), silica (medium) nanoparticles embedded in an epoxy (soft) matrix is provided in Figure 2a. Here it is not possible to see the fine features in the crystalline gold and iron-oxide nanoparticles because of the aforementioned dose and contrast challenges. However, employing a smart two-pass acquisition strategy has been shown to improve signal to noise for these challenging samples while reducing overall dose.[7,8] Scanning electron microscopy techniques have the 
advantage that they can decouple acquisition parameters with spatial position, making it possible to apply different doses to different areas of the sample depending on the local beam sensitivity.

Figure 2 shows the workflow of such a strategy. Here, (a) an ADF image is acquired with a short dwell time to minimize damage. Using an open-source algorithm that is compatible with electron microscopy software, the image is (b) background subtracted and (c) segmented into components using an unsupervised k-means clustering algorithm in real time. This creates (d) a mask, which can be used to guide the probe for a second pass at data acquisition with adaptive dwell time. This on-the-fly dynamic strategy is compatible with acquisition of images, hyperspectral data, and 4D datasets.

This presentation will speak about the challenges associated with imaging hybrid structures and focus on emerging techniques for studying this important class of materials.[9]
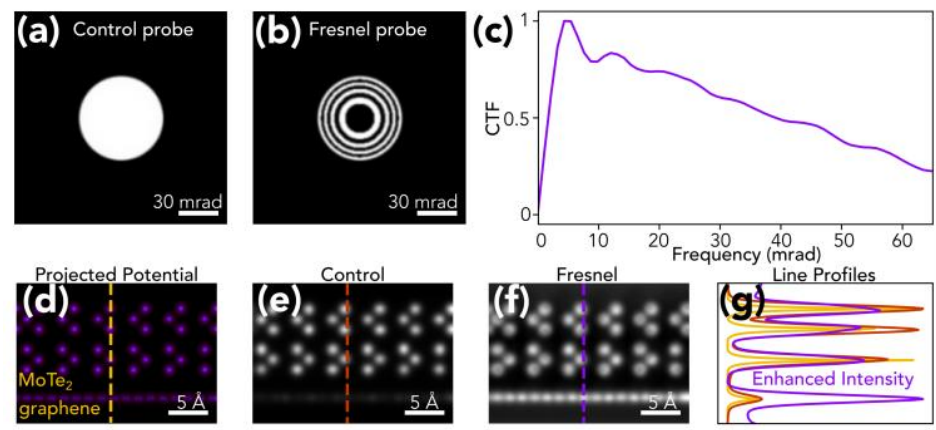

Figure 1. Phase plate study of a molybdenum ditelluride/graphene layered heterostructure: Phase of the (a) control and (b) Fresnel probe before sample interaction. (c) The contrast transfer function for the modified probe. (d) Projected potential of MoTe2/graphene sample. (e) Dark field (35-120 mrad) image of the area. (f) Image of same region with Fresnel probe, showing improved contrast between heavy and light elements. $(\mathrm{g})$ line profile comparing contrast from (d)-(f). Line color in (g) corresponds to color in (d)-(f).
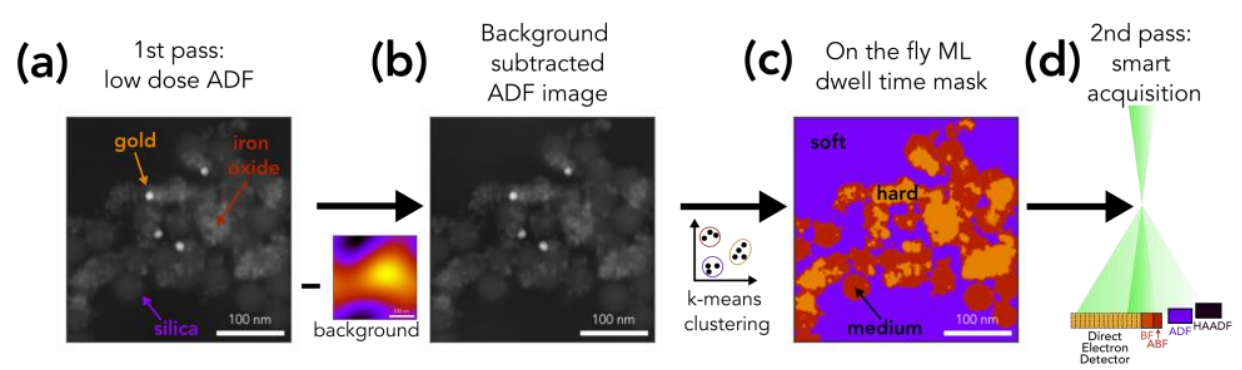

Figure 2. Proposed smart two-pass data acquisition workflow using example of gold (hard), iron oxide (hard), silica (medium) nanoparticles embedded in epoxy (soft) matrix: (a) an ADF image can be acquired, which is subsequently (b) background subtracted. Using a k-means clustering algorithm, the image can be segmented into hard, medium, and soft components, creating a (c) dwell time mask. This mask can be used to direct the beam time for real-time adaptive sampling to reduce the overall dose. 
References

[1] Wang, Da-Wei, et al. ACS nano 3.7 (2009): 1745-1752.

[2] Lin, Qing-Yuan, et al. Science 359.6376 (2018): 669-672.

[3] Gong, Shu, et al. Advanced Materials 32.15 (2020): 1902278.

[4] Ribet, Stephanie M., Murthy, Akshay A., et al., Materials Today

(2021), https://doi.org/10.1016/j.mattod.2021.05.006

[5] Ophus, Colin, et al. Nature communications 7.1 (2016): 1-7.

[6] Tomita, Masato, et al. Ultramicroscopy 218 (2020): 113089.

[7] Hujsak, Karl A., et al. Micron 108 (2018): 31-40.

[8] Dahmen, Tim, et al. Scientific reports 6.1 (2016): 1-11.

[9] This material is based upon work supported by the National Science Foundation under Grant No. DMR-1929356. This work made use of the Electron Probe Instrumentation Center (EPIC) and BioCryo facilities of Northwestern University's NUANCE Center, which has received support from the Soft and Hybrid Nanotechnology Experimental (SHyNE) Resource (NSF ECCS-2025633), the IIN, and Northwestern's MRSEC program (NSF DMR-1720139). This work was supported by Air Force Research Laboratory grant FA8650-15-2-5518, and partially supported by Air Force Office of Scientific Research award number FA9550-17-1-0348 and Army Research Office MURI grant W911NF1810200. Research reported in this publication was supported in part by instrumentation provided by the Office of The Director, National Institutes of Health of the National Institutes of Health under Award Number S10OD026871. The content is solely the responsibility of the authors and does not necessarily represent the official views of the National Institutes of Health. This research was supported in part through the computational resources and staff contributions provided for the Quest high performance computing facility at Northwestern University which is jointly supported by the Office of the Provost, the Office for Research, and Northwestern University Information Technology. 